\title{
Choisir avec soin (et sensément)
}

lusieurs associations médicales ont fait front commun avec l'Association médicale canadienne (AMC) pour donner le coup d'envoi de la campagne Choisir avec soin (www.ChoisirAvecSoin.org). Deux associations du domaine chirurgical étaient au nombre des premiers participants, nommément l'Association canadienne des chirurgiens généraux (ACCG) et l'Association canadienne d'orthopédie (ACO). L'initiative Choisir avec soin vise à encourager le dialogue entre les médecins et les patients au sujet de la pertinence des examens médicaux, des traitements et des interventions.

La campagne Choisir avec soin semble être une bonne initiative pour promouvoir une plateforme nationale de communication sur les soins de santé au Canada. Le gouvernement fédéral s'est retiré du domaine des soins de santé et a laissé un vide au sommet. Les paiements de transfert en matière de santé ont de moins en moins de valeur et ne représentent plus qu'une petite partie de l'ensemble des dépenses de santé à l'échelle nationale. Un manque de gestion nationale des données dans les grands postes de dépenses comme les soins de traumatologie et d'autres efforts montrent que le gouvernement fédéral laisse le domaine médical sans leadership politique clair. Nous devons dorénavant tenir un dialogue plus constructif et continu concernant tous les soins de santé. L'initiative Choisir avec soin semble être une bonne façon de commencer.

Pour encourager la communication entre les patients et les médecins, nous devrons éviter de nous quereller et de nous pointer du doigt les uns les autres quand nous choisissons quels actes médicaux devraient être examinés. Vu l'enveloppe fermée de financement de la santé, il sera très tentant pour certaines spécialités de regarder le morceau du gâteau des autres groupes et de dire que nous devrions réduire leurs dépenses. Bien que certaines de ces observations puissent être justes, ce n'est toutefois pas la raison d'être de Choisir avec soin. Nos voisins du Sud ont déjà entrepris de faire ces « choix judicieux », mais bon nombre des groupes médicaux ont choisi de désigner comme inappropriées des interventions d'autres spécialités et de minimiser leur propre besoin d'introspection. Ce n'était certes pas l'approche imaginée pour Choisir avec soin. Cependant, il se pourrait que ce soit aussi la voie que l'initiative empruntera au Canada, ce qui ne serait pas nécessairement une mauvaise idée, si elle permettait d'ouvrir une communication constructive entre les spécialités concernant l'examen des processus en soins de santé.

L'autre difficulté, c'est que même si Choisir avec soin cherche à améliorer la communication et l'information, les commentaires formulés au sein du groupe pourraient être utilisés par les administrations provinciales pour limiter les actes médicaux, une répercussion qui ne serait pas souhaitable. Ce n'est pas au gouvernement, en tant que propriétaire souvent absent, de déterminer quels actes médicaux les médecins peuvent effectuer. Cela devrait relever de façon non équivoque des gestionnaires de l'éducation médicale continue au sein de chaque société nationale de spécialité et organisation provinciale ou territoriale des médecins. Il appartient aux gestionnaires des programmes respectifs de formation médicale des organisations de leadership, telles que l'ACO et l'ACCG, de faire la liste des traitements pertinents. Il y a en effet des indications limitées pour certaines interventions, mais c'est au médecin traitant de prendre la décision, car il est la personne qui connaît le mieux les indications pour les actes médicaux.

Linitiative Choisir avec soin nous aidera à entamer un dialogue - et ce dialogue ne doit pas se tenir entre les sociétés médicales dissidentes ou les administrations provinciales de la santé, mais plutôt entre les médecins et leurs patients. Nous devons être prudents dans la mise en œuvre de cette initiative, afin d'obtenir réellement ce résultat.

\section{Edward J. Harvey, MD \\ Co-rédacteur, Fournal canadien de chirurgie}

Intérêts concurrents : Aucuns déclarés.

DOI: $10.1503 /$ cjs.005414 